\title{
ASSOCIATION OF INSULIN RESISTANCE LINKED DISEASES AND HAIR LOSS IN ELDERLY MEN. FINNISH POPULATION-BASED STUDY
}

\author{
Päivi Hirsso ${ }^{1}$, Mauri Laakso ${ }^{1,2}$, Veikko Matilainen ${ }^{1}$, Liisa Hiltunen ${ }^{1,2,3}$, Ulla Rajala ${ }^{1}$, Jari Jokelainen ${ }^{1,2}$, \\ Sirkka Keinänen-Kiukaanniemi ${ }^{1,3}$ \\ ${ }^{1}$ University of Oulu, Department of Public Health Science and General Practice, University of Oulu, Finland \\ 2Unit of General Practice, Oulu University Hospital, Finland \\ ${ }^{3}$ Oulu Health Center, City of Oulu, Finland
}

\begin{abstract}
SUMMARY
Previous investigations have shown an association of androgenetic alopecia (AGA) with insulin resistance related disorders such as ischemic heart disease. An association between AGA and anthropometric abnormalities linked with insulin resistance and heredity in women aged 63 years has also been shown. We therefore compared 63-year-old men with AGA and ones with normal hair status for insulin resistance linked parameters.

A population of 245 men aged 63 years, who were participants in a population-based cross-sectional study in the City of Oulu, underwent a medical check-up including assessment of hair status on the Hamilton-Norwood scale and determination of anthropometric measures, blood pressure, fasting glucose and serum lipids.

Fifty eight per cent of the men reported extensive hair loss (grade III-VII). Hypertension and the use of antihypertensive drugs were common among men with AGA ( $61 \%$ vs. $45 \%$ and $50 \%$ vs. $26 \%$, respectively). The rates of diabetes and hyperinsulinemia ( $21 \%$ vs. $12 \%$ and $61 \%$ vs. $49 \%$ ) were higher among men with AGA compared to those with normal hair status but no difference was seen in other factors.

Our findings show that AGA is common among Finnish men aged 63 years but that it is also associated with insulin linked disturbances, such as hypertension and diabetes. Such men developing AGA might benefit from attention in medical check-up.
\end{abstract}

Key words: androgenetic alopecia, hair loss, insulin resistance

Address for correspondence: P. Hirsso, BOX 5000, Department of Public Health Science and General Practice, University of Oulu, FIN-900014 University of Oulu, Finland. E-mail: paivi.hirsso@oulu.fi

\section{INTRODUCTION}

An association between androgenetic alopecia (AGA) and cardiovascular events such as myocardial infarction and ischemic heart disease has been previously reported (1-5). Finnish case-control studies have suggested an increased risk of hyperinsulinaemia and insulin resistance related disorders such as hypertension and dyslipidaemia and of severe coronary heart disease among men with early onset AGA compared to men with normal hair status (6-7). Associations between female AGA (grade II or III on Ludwig`s scale) among 63-year-old women and some markers of insulin resistance, android-type obesity and heredity have been reported recently (8). The aim of the present study was to test the association of male-pattern AGA in men aged 63 with insulin resistance-linked disorders.

\section{SUBJECTS AND METHODS}

This study was a population-based cross-sectional study. All the participants $(\mathrm{n}=831)$ of a previous population-based followup study carried out in 1990-1992 (9) in the City of Oulu were invited to participate in this study in 1996-1998, and 593 of them participated (245 men). Questionnaires, interviews, clinical exa- minations and laboratory tests were used to collect data during the follow-up period in 1996-1998. The material and methods have been described in more detail in previous publications $(8,10)$.

The hair status of 221 men was assessed by a trained nurse as part of the clinical examination using the Hamilton-Norwood scale (11). The original classes I-II were merged form to larger categories: normal hair/mild alopecia (classes I-IIIb) and extensive hair loss (classes IIIvertex-VII). Additional questions asked about the most plentiful hair loss if any significant had existed and the family history of hair loss also.

The potential insulin resistance linked factors; body mass index (BMI, weight in kilograms divided by the square of height in meters), neck/hip and waist circumference, and systolic and diastolic blood pressure were measured. Height and weight in light clothing for calculating BMI were measured in the clinical examination. Two measurements of blood pressure (BP) were made by the physician from both arms in sitting and recumbent positions. The mean value of these four measurements was used in analyses. Hypertension was defined as either a systolic blood pressure $\geq 160 \mathrm{mmHg}$ or a diastolic blood pressure $\geq 90 \mathrm{mmHg}$ or being on antihypertensive medication regardless of the blood pressure values. Drug treatment for hypertension was recorded in an interview conducted by a physician. In addition to this, the postal questionnaire included the following questions: "Do you 
have diabetes mellitus/hypertension diagnosed by a physician?" and "Do you use any antidiabetic medication?" Smoking habits were asked in the postal questionnaire.

The following biochemical data concerning the traditional cardiovascular risk factors were recorded: fasting total cholesterol (chol), high-density lipoprotein (HDL)-cholesterol, triglyceride (TG) and insulin concentrations as well as fasting and two-hour glucose concentrations after a $75 \mathrm{~g}$ glucose load measured from a blood-sample taken after an overnight $(10 \mathrm{~h})$ fast. A standardized $75 \mathrm{~g}$ oral glucose tolerance test (OGTT) was performed according to the instructions of the WHO Study Group (12). Subjects with impaired glucose tolerance and normal glucose tolerance were combined as non-diabetic subjects. Serum immunoreactive insulin concentrations were measured by RIA using the Phadeseph Insulin RIA 100 kit (Pharmacia Diagnostics AB, Uppsala, Sweden), which also detects proinsulin and proinsulin conversion products with considerable sensitivity. The cross-reactivity of proinsulin in this assay is about $41 \%$. Insulin levels were not analysed from the samples of the diabetic patients with insulin treatment. Urine albumin and creatine concentrations were measured from an overnight spot urine sample. The highest decile of the urinary albumin to creatine ratio $(\geq 2.5 \mathrm{mg} / \mathrm{mmol})$ was used as a measure of microalbuminuria. To measure insulin sensitivity, an insulin sensitivity check index (Quicki) was used (13-14). The determinations mentioned above were made in the laboratory with a standardized quality control system.

Table 1. Means/medians and standard deviations (SD) / interquartile range of background characteristics among men aged 63 years with extensive hair loss and normal hair

\begin{tabular}{|l|c|c|c|c|}
\hline & \multicolumn{2}{|c|}{$\begin{array}{c}\text { Extensive hair loss } \\
\mathrm{n}=128\end{array}$} & \multicolumn{2}{c|}{$\begin{array}{c}\text { Normal hair } \\
\mathrm{n}=92\end{array}$} \\
& Mean & Sd & Mean & Sd \\
\hline Weight (kg) & 82.9 & 12.6 & 85.5 & 12.6 \\
\hline Length (cm) & 173.0 & 5.6 & 174.6 & 6.3 \\
\hline BMl (kg/m²) & 27.6 & 3.6 & 27.9 & 3.7 \\
\hline Neck circ. (cm) & 40.7 & 2.6 & 41.1 & 2.6 \\
\hline Hip circ. (cm) & 101.4 & 7.1 & 102.4 & 7.2 \\
\hline Waist circ. (cm) & 95.8 & 10.7 & 97.3 & 9.9 \\
\hline WHR & 0.94 & 0.06 & 0.95 & 0.05 \\
\hline SBP (mmHg) & 142.8 & 18.5 & 141.9 & 18.3 \\
\hline DBP (mmHg) & 79.3 & 8.0 & 79.4 & 6.9 \\
\hline Fs-chol (mmol/l) & 5.7 & 0.9 & 5.6 & 0.8 \\
\hline $\begin{array}{l}\text { HDL-chol } \\
\text { (mmol/l) }\end{array}$ & 1.3 & 0.3 & 1.3 & 0.3 \\
\hline $\begin{array}{l}\text { 2-h gluc } \\
\text { (mmol/l) }\end{array}$ & 6.9 & 2.2 & 6.8 & 2.1 \\
\hline & Median & Q1-Q3 & Median & Q1-Q3 \\
\hline $\begin{array}{l}\text { Fs-trigly } \\
\text { (mmol/l) }\end{array}$ & 1.3 & $0.9-1.7$ & 1.2 & $0.9-1.8$ \\
\hline Fb-gluk (mmol/l) & 4.9 & $4.6-5.6$ & 5.0 & $4.8-5.5$ \\
\hline Fs-ins (mU/l) & 10.0 & $8.0-15.0$ & 9.0 & $8.0-14.0$ \\
\hline $\begin{array}{l}\text { U-alb/krea ratio } \\
\text { Insulin sensitivity } \\
\text { (Quicki) }\end{array}$ & 0.92 & $0.68-1.18$ & 0.91 & $0.71-1.54$ \\
\hline & 0.33 & $0.32-0.35$ & 0.34 & $0.32-0.35$ \\
\hline
\end{tabular}

${ }^{*} \mathrm{p}=0.021$ (t-test)

\section{Statistical Analyses}

The summary statistics for normally distributed continuous variables were expressed as mean and standard deviation and as median with interquartile range (25th and 75 th percentiles) for non-normally distributed variables. The statistical differences between the continuous variables were tested by the t-test when the distribution was normal and with the Mann-Whitney U-test in a non-normal situation. For categorical variables, the $\chi^{2}$ test was used to calculate the unadjusted odds rations (OR) and their 95\% confidence intervals $(\mathrm{CI})$ for each dichotomized variable. On the basis of bivariate logistic regression analysis, adjustments were performed only for the potential risk factors that were significantly associated with hair loss. Statistical analyses were performed using SPSS for Windows (version 10.0)

\section{RESULTS}

The prevalence of extensive hair loss was $58.4 \%$ among these 63 -year-old men. Twenty-two per cent of the men with extensive hair loss reported the onset of abundant hair loss at an age younger than 35 years. The means or medians of the continuous variables relative to hair status are presented in Table 1 . The men with extensive hair loss were shorter $(173 \mathrm{~cm}$ vs. $175 \mathrm{~cm}, \mathrm{p}=0.021)$ but their body weight was lower compared to those with normal hair status, while no difference in BMI or the other biochemical markers of insulin resistance was seen.

The absolute numbers and percentages of insulin resistance associated states, such as diabetes, dyslipidemia and hypertension as well as hyperinsulinemia and microalbuminuria, smoking status and the presence of maternal or paternal hair loss in the subgroups of hair status classes are presented in Table 2. Adjustment for hyperinsulinemia, hypertension, diabetes and paternal heredity did not alter these associations (Table 3). The use of antihypertensive medication was clearly more frequent among the men with extensive hair loss $(50 \%$ vs. $26 \%, \mathrm{p}=0.0003)$ and those with extensive hair loss used more frequently $\beta$-channel blockers $(30 \%$ vs. $15 \%, p=0.010)$ and angiotensin-converting enzyme

Table 2. Percentages of insulin resistance associated disease, hyperinsulinemia, microalbuminuria, smoking and heredity among men aged 63 years with extensive hair loss and normal hair

\begin{tabular}{|c|c|c|c|c|c|}
\hline & \multicolumn{2}{|c|}{$\begin{array}{c}\text { Extensive hair loss } \\
n=129\end{array}$} & \multicolumn{2}{|c|}{$\begin{array}{c}\text { Normal hair } \\
n=92\end{array}$} & \multirow[b]{2}{*}{$p$} \\
\hline & $\mathrm{n}$ & $\%$ & $n$ & $\%$ & \\
\hline Hyperinsulinemia ${ }^{1)}$ & 78 & 61 & 44 & 49 & 0.078 \\
\hline Diabetes ${ }^{2)}$ & 27 & 21 & 11 & 12 & 0.081 \\
\hline Dyslipidemia ${ }^{3)}$ & 45 & 35 & 34 & 37 & ns. \\
\hline Hypertension ${ }^{4)}$ & 77 & 62 & 41 & 45 & 0.016 \\
\hline Microalbuminurias) & 11 & 9 & 7 & 8 & ns. \\
\hline Paternal heridity & 57 & 47 & 21 & 23 & 0.0003 \\
\hline Maternal heredity & 3 & 3 & 1 & 1 & ns. \\
\hline Smoking & 25 & 19 & 19 & 21 & ns. \\
\hline
\end{tabular}

${ }^{1)}$ Hyperinsulinemia: fs-insulin $\geq 10$ U/I. ${ }^{2}$ Diabetes: according to WHO 1997 criteria or antidiabetic drugs. ${ }^{3}$ Dyslipidemia: hypertriglyceridemia $\geq 1.7 \mathrm{mmol} / \mathrm{l}$, HDL-chol $<1.0 \mathrm{mmol} / \mathrm{l}$ or lipid-lowering agents. ${ }^{4)}$ Hypertension: systolic blood pressure $\geq 160 \mathrm{mmHg}$ or diastolic blood pressure $\geq 90 \mathrm{mmHg}$ or antihypertensive drugs. ${ }^{5}$ Microalbuminuria: urinary albumin-to-serum creatinine ratio $\geq 2.5$ 
Table 3. Odds ratios (OR) and $95 \%$ confidence intervals (95\% $\mathrm{Cl}$ ) of extensive hair loss associated with hyperinsulinemia, diabetes, hypertension and paternal heredity in men

\begin{tabular}{|l|c|c|c|c|}
\hline & \multicolumn{2}{|c|}{ Unadjusted } & \multicolumn{2}{c|}{ Adjusted } \\
\hline & OR & $95 \% \mathrm{Cl}$ & OR & $95 \% \mathrm{Cl}$ \\
\hline Hyperinsulinemia') $^{1)}$ & 1.63 & $0.95-2.81$ & 1.56 & $0.85-5.53$ \\
\hline Diabetes $^{2)}$ & 1.96 & $0.92-4.20$ & 1.94 & $0.82-4.59$ \\
\hline Hypertension $^{3)}$ & 1.96 & $1.13-3.38$ & 1.81 & $0.97-3.37$ \\
\hline Paternal heredity $^{3}$ & 3.01 & $1.65-5.51$ & 2.71 & $1.44-5.13$ \\
\hline
\end{tabular}

${ }^{1)}$ Hyperinsulinemia: fs-insulin $\geq 10 \mathrm{U} / \mathrm{l}$

2)Diabetes: according to WHO 1997 criteria or antidiabetic drugs.

${ }^{3}$ Hypertension: systolic blood pressure $\geq 160 \mathrm{mmHg}$ or diastolic blood pressure $\geq 90 \mathrm{mmHg}$ or antihypertensive drugs

inhibitors (ACE) $(17 \%$ vs. $7 \%, \mathrm{p}=0.020)$ compared to the men with normal hair status.

\section{DISCUSSION}

This population-based study revealed an association of AGA with insulin resistance linked diseases among men aged 63 years, but associations between AGA and markers of insulin resistance were rare. Previous investigations have suggested a link between AGA and elevated BMI in older men (1, 6-7), but some studies have given negative results $(5,15-17)$. In one population-based study (15) the preliminary association between AGA and cardiovascular risk factors disappeared after adjustment for age. In that study, the final classification of AGA was based on self-reported hair status and the age distribution of study population differs from those used in our study of 63 years old men. Lotufo et al. (5) and Trevisan et al. (17), who focused on selected populations, found higher BP and higher concentration of serum cholesterol among men with AGA. A Finnish case-control study suggests that AGA is not associated only with cardiovascular risk factors (6) but also with severe coronary heart disease (7).

In this study, the prevalence of hair loss was $58 \%$ among northern Finnish men aged 63 years. The prevalence of malepattern hair loss is known to increase with age. For example, the occurrence of extensive hair loss has been reported to be $40 \%$ in subjects aged $40-49$ years, $50 \%$ in subjects aged 50-59 years, and $60 \%$ in subjects aged $60-69$ years (11). In the Framingham study hair loss in men aged 55-64 years amounted to $68 \%$ (3), which results are in accordance with our findings. The recent studies from Australia (16) and Singapore (18) both reported higher prevalence of AGA in men of this age. There are ethnic differences in the study populations and the classification of AGA had been modified by authors. In a Korean study, the prevalence of AGA in men aged 60-69 years was 34\% (19) which is lower than among Caucasian men.

As previously reported in women in our study (8), paternal heredity was also associated with AGA in men. The association was stronger than earlier reported in Korean men (19) and as we know, the heritability and genetic background of hair loss have been under consideration for decades (20). Hair loss in female relatives was rarely reported, which could be partly due to the difficulty to recognize female-type hair loss. The most intensive hair loss starting at an age younger than 35 years in $22 \%$ of men with hair loss was quite low. This might be due to the memorybias or misunderstanding of the question.

The strengths of our study include the population-based sample and the standard classification scale used by trained nurses. The Hamilton-Norwood scale is considered to be well reproducible (4). One limitation is that we could not determine the effect of antihypertensive drugs on AGA. According the previous studies, antihypertensive drugs ( $\alpha$ - and $\beta$-channel blockers, ACE, calcium channel blockers) may induce hair loss (21-22) and, on the other hand, men with extensive hair loss used more frequently $\beta$-channel blockers or ACEs than men with normal hair.

To sum up, AGA seems to connect with insulin resistance linked diseases in men at older age but not with the markers of insulin resistance. It remains unclear whether insulin resistance has led to development of the disease because of aging among subjects with AGA or whether there are some connections between the use of drugs and AGA. In a retrospective study of this kind, the effects of drugs on hair status and the impacts of health education given to balding men with diabetes and hypertension cannot be excluded. The risk of AGA increases with age in general, and regardless of diseases or medication, there is also a tendency to hair loss in the healthy population and such men developing AGA might benefit from attention in medical check-up.

\section{REFERENCES}

1. Ford ES, Freedman DS, Byers T. Baldness and ischemic heart disease in a national sample of men. Am J Epidemiol. 1996 Apr 1;143(7):651-7.

2. Hambly RI, Aintablian A, Hoffman I, Kramer J, Marx JW. Is male baldness a coronary risk factor? Clin Res. 1977;25(3):226A.

3. Herrera CR, D“Agostino RB, Gerstman BB, Bosco LA, Belanger AJ. Baldness and coronary heart disease rates in men from the Framingham Study. Am J Epidemiol. 1995 Oct 15;142(8):828-33.

4. Lesco S, Rosenberg L, Shapiro S. A case-control study of baldness in relation to myocardial infarction in men. JAMA. 1993 Feb 24;269(8):998 1003. Erratum in: JAMA 1993 May 19;269(19):2508.

5. Lotufo PA, Chae CU, Ajani UA, Hennekens CH, Manson JE. Male pattern baldness and coronary heart disease: the Physicians' Health Study. Arch Intern Med. 2000 Jan 24;160(2):165-71.

6. Matilainen V, Koskela P, Keinanen-Kiukaanniemi S. Early androgenetic alopecia as a marker of insulin resistance. Lancet. 2000 Sep 30;356(9236):1165-6.

7. Matilainen VA, Makinen PK, Keinanen-Kiukaanniemi SM. Early onset of androgenetic alopecia associated with early severe coronary heart disease: a population-based, case-control study. J Cardiovasc Risk. 2001 Jun;8(3):147-51.

8. Matilainen V, Laakso M, Hirsso P, Koskela P, Rajala U, KeinanenKiukaanniemi S. Hair loss, insulin resistance, and heredity in middleaged women. A population-based study. J Cardiovasc Risk. 2003 Jun;10(3):227-31.

9. Rajala U, Keinanen-Kiukaanniemi S, Uusimaki A, Reijula K, Kivela SL. Prevalence of diabetes mellitus and impaired glucose tolerance in a middle-aged Finnish population. Scand J Prim Health Care. 1995 Sep;13(3):222-8.

10. Rajala U, Koskela P, Keinanen-Kiukaanniemi S. Hyperglycemia as a risk factor of mortality in a middle-aged Finnish population. J Clin Epidemiol. 2001 May;54(5):470-4.

11. Norwood OT. Male pattern baldness: classification and incidence. South Med J. 1975 Nov;68(11):1359-65.

12. World Health Organization. Diabetes mellitus: report of a WHO Study Group. Geneva: WHO; 1985. WHO Technical Report Series No. 727.

13. Katz A, Nambi SS, Mather K, Baron AD, Follmann DA, Sullivan G, et al. Quantitative insulin sensitivity check index: a simple, accurate method for assessing insulin sensitivity in humans. J Clin Endocrinol Metab. 2000 Jul;85(7):2402-10.

14. Hrebíček J, Janout V, Malinčíková J, Horáková D, Čížek L. Detection of insulin resistance by simple quantitative insulin sensitivity check 
index QUICKI for epidemiological assessment and prevention. J Clin Endocrinol Metab. 2002 Jan;87(1):144-7.

15. Ellis JA, Stebbing M, Harrap SB. Male pattern baldness is not associate with established cardiovascular risk factors in the general population. Clin Sci (Lond). 2001 Apr;100(4):401-4.

16. Severi G, Sinclair R, Hopper JL, English DR, McCredie MR, Boyle P, et al. Androgenetic alopecia in men aged 40-69 years: prevalence and risk factors. Br J Dermatol. 2003 Dec;149(6):1207-13.

17. Trevisan M, Farinaro E, Krogh V, Jossa F, Giumetti D, Fusco G, et al. Baldness and coronary heart disease risk factors. J Clin Epidemiol. 1993 Oct;46(10):1213-8.

18. Tang PH, Chia HP, Cheong LL, Koh D. A community study of male androgenetic alopecia in Bishan, Singapore. Singapore Med J. 2000 May;41(5):202-5.
19. Paik JH, Yoon JB, Sim WY, Kim BS, Kim NI. The prevalence and types of androgenetic alopecia in Korean men and women. Br J Dermatol. 2001 Jul;145(1):95-9.

20. Kuster W, Happle R. The inheritance of common baldness: two B or not two B? J Am Acad Dermatol. 1984 Nov;11(5 Pt 1):921-6.

21. Tosi A, Misciali C, Piraccini BM, Peluso AM, Bardazzi F. Drug-induced hair loss and hair growth. Incidence, management and avoidance. Drug Saf. 1994 Apr;10(4):310-7.

22. Whiting D, Howsden FL. Color atlas of differential diagnosis of hair loss. Fairfield: Canfield Publishing; 1998.

Received May 3, 2005 Accepted November 14, 2005 